\title{
Editorial
}

\section{Tackling the Biological Diversity in Early Triple-Negative Breast Cancer}

\author{
Mafalda Oliveira ${ }^{a, b} \quad$ Cristina Saura ${ }^{a, b}$ \\ a Department of Medical Oncology, Vall d'Hebron Hospital Universitari, Vall d'Hebron Barcelona Hospital Campus, \\ Barcelona, Spain; 'breast Cancer and Melanoma Group, Vall d'Hebron Institute of Oncology, Vall d'Hebron \\ Barcelona Hospital Campus, Barcelona, Spain
}

Triple-negative breast cancer (TNBC) accounts for approximately $10-15 \%$ of all breast cancers and is associated with worse prognosis when compared to other subtypes [1]. However, prognosis in not the same across different TNBC subtypes, and a better understanding of TNBC biology is fundamental to better tailor treatment, in order to maximize patient benefit without compromising quality of life.

Understanding the biology of TNBC is fundamental to acknowledge the deep challenges that patients and caregivers face when choosing the most adequate therapy. In this monograph, Herrera Juarez et al. [2] describe TNBC biology and classification focused on immunohistochemistry, transcriptomics, genomics features, and the new immune biomarkers. They highlight the inherent challenge related to TNBC definition, encompassing heterogenous molecular and immunological entities with a variety of clinical phenotypes.

While there are some biomarkers that have already been included in clinical practice for clinical decision-making, others remain investigational. Patients with PD-L1-positive tumors may benefit more from checkpoint inhibitor-based approaches, at least in the metastatic setting [3], and for this reason should be tested for PD-L1 before first-line treatment for metastatic disease. Olaparib and talazoparib are FDA- and EMA-approved for the treatment of metastatic HER2-negative breast cancer in patients with a germline BRCA1 and/or BRCA2 mutation [4, 5], and consequently testing for germline mutations should also be considered in this setting. The predictive values of other biomarkers for selection of patients, such as alterations in the PI3K pathway (PI3KCA mutations, AKT1 mutations, PTEN genomic alterations), are currently being tested in clinical trials. Another important issue is prioritization of the biomarker information to choose the most adequate drug and drug sequencing. For instance, it is currently unknown whether a patient with a germline BRCA1/2 mutation and a metastatic PD-L1-positive TNBC should be offered a PARP inhibitor or a checkpoint inhibitor in the first-line setting. Our personal approach would be to favor the drug or combination with the highest level of clinical evidence of benefit. To make things even more complex, there are trials combining PARP inhibitors with checkpoint inhibitors, and these results will definitely be informative to future treatment decisions. Whether these biomarkers will be as important in clinical decision-making in the early setting remains to be fully elucidated, and issues related to tissue optimization use for biomarker determination will need to be addressed in the future.

In the second paper of this monograph, Furlanetto and Loibl [6] review the current and future treatment approaches in early TNBC. Curative treatment for TNBC includes a combination of surgery, radiotherapy, and systemic therapy, mainly using chemotherapy either before (neoadjuvant) and/or after surgery (adjuvant). One important controversy in this setting is the use of platinum salts as part of the neoadjuvant regimen for these patients. Results from the randomized trials suggest that patients that receive neoadjuvant carboplatin may have higher response rates and pathological complete response rates compared to those that do not $[7,8]$. However, long-term outcome results differ among trials. Furthermore, the addition of carboplatin to standard anthracycline- and taxane-based neoadjuvant che- 
motherapy may have important tolerability issues leading to significant discontinuation rates. In this setting, it seems clear that predictive biomarkers would play an important role in defining the best treatment options for patients with early-stage TNBC. Of note, patients with good-prognosis features, such as high tumor infiltrating lymphocytes, have a good long-term prognosis and may be suitable candidates to consider de-escalation strategies [9].

Another area of unmet need in the early setting is the presence of residual disease after standard neoadjuvant therapy. These patients have a high risk of relapse and may benefit from escalation treatment strategies with new drugs and combinations, in a setting where capecitabine is now widely used after the publication of the Create-X trial [10]. However, not all patients with residual disease recur, even in the absence of capecitabine; on the other hand, there are still patients receiving capecitabine who experience relapse of their disease. Research in this field using biomarker-guided strategies to target residual disease may help improve the prognosis of this specific group of patients in the future.

Further stratification of prognosis can be achieved with the incorporation of novel biomarkers, such as circulating tumor DNA (ctDNA) detection, after a curative treatment has been delivered [11]. In the c-TRAK TN trial (NCT03145961), for instance, patients with TNBC are monitored for the emergence of ctDNA during the first year after ending their curative treatment. If ctDNA becomes apparent, and in the absence of clinical or radiological relapse, patients are randomized to start treatment with pembrolizumab or to continue standard clinical follow-up.
In conclusion, a deep understanding of the biology and the drivers of tumor progression is key to better tailoring treatments in TNBC, especially in the early setting, in order to increase the cure rates of these patients.

\section{Disclosure Statement}

M. Oliveira: Institutional financial interests: AstraZeneca, Philips, Genentech, Roche, Seattle Genetics, Zenith Epigenetics, GSK, Immunomedics, Novartis, Boehringer, and Puma. Personal financial interests (advisory and/or speaker): Roche, Novartis, GSK, Seattle Genetics, Puma, and AstraZeneca. Member of the Executive Board of the SOLTI Breast Cancer Group.

C. Saura: Personal financial interests: Served as consultant, participated in advisory boards, or received travel grants from AstraZeneca, Celgene, Daiichi Sankyo, F. Hoffmann-La Roche Ltd., Genomic Health, Merck, Sharp and Dhome España S.A., Novartis, Odonate Therapeutics, Pfizer, Philips Healthwork, Pierre Fabre, prIME Oncology, Puma, Synthon, and Sanofi Aventis. Institutional financial interests: Paid directly to her Institution: AstraZeneca, Daiichi Sankyo, Eli Lilly and Company, Genentech, Immunomedics, Macrogenics, Merck, Sharp and Dhome España S.A., Novartis, Pfizer, Piqur Therapeutics, Puma, Roche, Synthon, and Zenith Pharma. Member of the Scientific Committee of SOLTI (Academic Research Group in Breast Cancer). Member of the ESMO Faculty (2017-2021).

\section{Funding Sources}

No funding is declared.

\section{Author Contributions}

Both authors contributed to the conception, design, analysis, and interpretation of the data, drafted the work, gave their final approval of the version to be published, and agree to be accountable for all aspects of the work.

\section{References}

1 Dawson SJ, Provenzano E, Caldas C. Triple negative breast cancers: clinical and prognostic implications. Eur J Cancer. 2009 Sep; 45(Suppl 1):27-40.

2 Herrera Juarez M, Tolosa Ortega P, Sanchez de Torre A, Ciruelos Gil E. Biology of the Triple-Negative Breast Cancer: Immunohistochemical, RNA, and DNA Features. Breast Care (Basel). doi: 10.1159/000508758 [Epub ahead of print].

3 Schmid P, Adams S, Rugo HS, Schneeweiss A, Barrios $\mathrm{CH}$, Iwata $\mathrm{H}$, et al.; IMpassion 130 Trial Investigators. Atezolizumab and Nab-Paclitaxel in Advanced Triple-Negative Breast Cancer. N Engl J Med. 2018 Nov;379(22): 2108-21.

4 Robson M, Im SA, Senkus E, Xu B, Domchek SM, Masuda N, et al. Olaparib for Metastatic Breast Cancer in Patients with a Germline BRCA Mutation. N Engl J Med. 2017 Aug; 377(6):523-33.
5 Litton JK, Rugo HS, Ettl J, Hurvitz SA, Gonçalves A, Lee KH, et al. Talazoparib in Patients with Advanced Breast Cancer and a Germline BRCA Mutation. N Engl J Med. 2018 Aug; 379(8):753-63.

6 Furlanetto J, Loibl S. Optimal Systemic Treatment for Early Triple-Negative Breast Cancer. Breast Care (Basel). doi: 10.1159/ 000508759 [Epub ahead of print].

7 von Minckwitz G, Schneeweiss A, Loibl S, Salat C, Denkert C, Rezai M, et al. Neoadjuvant carboplatin in patients with triple-negative and HER2-positive early breast cancer (GeparSixto; GBG 66): a randomised phase 2 trial. Lancet Oncol. 2014 Jun;15(7):74756.

8 Sikov WM, Berry DA, Perou CM, Singh B, Cirrincione CT, Tolaney SM, et al. Impact of the addition of carboplatin and/or bevacizumab to neoadjuvant once-per-week paclitaxel followed by dose-dense doxorubi- cin and cyclophosphamide on pathologic complete response rates in stage II to III triple-negative breast cancer: CALGB 40603 (Alliance). J Clin Oncol. 2015 Jan;33(1):1321.

9 Loi S, Drubay D, Adams S, Pruneri G, Francis PA, Lacroix-Triki M, et al. Tumor-Infiltrating Lymphocytes and Prognosis: A Pooled Individual Patient Analysis of Early-Stage TripleNegative Breast Cancers. J Clin Oncol. 2019 Mar;37(7):559-69.

10 Masuda N, Lee SJ, Ohtani S, Im YH, Lee ES, Yokota I, et al. Adjuvant Capecitabine for Breast Cancer after Preoperative Chemotherapy. N Engl J Med. 2017 Jun;376(22):214759.

11 Garcia-Murillas I, Chopra N, Comino-Méndez I, Beaney M, Tovey H, Cutts RJ, et al. Assessment of Molecular Relapse Detection in Early-Stage Breast Cancer. JAMA Oncol. 2019 Aug;5(10):1473. 